\title{
Present status of the USR project
}

\author{
C. P. Welsch • J. Harasimowicz • K. U. Kühnel • \\ A. Papash • M. Putignano • P. Schmid • J. Ullrich
}

Published online: 29 August 2009

(C) The Author(s) 2009. This article is published with open access at Springerlink.com

\begin{abstract}
The Facility for Low-energy Antiproton and Ion Research (FLAIR) and a large part of the wide physics program decisively rely on new experimental techniques to cool and slow down antiprotons to $20 \mathrm{keV}$, in particular on the development of an ultra-low energy electrostatic storage ring (USR). The whole research program connected with anti-matter/matter interactions is only feasible if such a machine will be realized. For the USR to fulfil its key role in the FLAIR project, the development of novel and challenging methods and technologies is necessary: the combination of the electrostatic storage mode with a deceleration of the stored ions from 300 $\mathrm{keV}$ to $20 \mathrm{keV}$, electron cooling at all energies in both longitudinal and transverse phase-space, bunching of the stored beam to ultra-short pulses in the nanosecond regime and the development of an in-ring reaction microscope for antiproton-matter
\end{abstract}

C. P. Welsch (凶) - J. Harasimowicz · K. U. Kühnel · A. Papash · M. Putignano ·

P. Schmid · J. Ullirich

Max Planck Institute for Nuclear Physics, Saupfercheckweg 1, 69117 Heidelberg, Germany

e-mail: carsten.welsch@quasar-group.org

A. Papash

JINR, Joliot-Curie 6, 141980 Dubna, Moscow Region, Russia

C. P. Welsch · J. Harasimowicz · A. Papash · M. Putignano · P. Schmid

Gesellschaft für Schwerionenforschung, Planckstrasse 1, 64291 Darmstadt, Germany

C. P. Welsch

The Cockcroft Institute of Accelerator Science and Technology,

WA4 4AD, Warrington, UK

C. P. Welsch

Department of Physics, The University of Liverpool, Liverpool, L69 7ZE, UK 
rearrangement experiments. In this contribution, the present status of the USR project is summarized and the new machine lattice is presented.

Keywords Particle accelerators $\cdot$ Storage rings $\cdot$ Antiprotons $\cdot$ Beam cooling

\section{Introduction}

Antiprotons stored and cooled at low energies in a storage ring or at rest in traps are highly desirable for the investigation of a large number of basic questions on fundamental interactions, on the static structure of exotic antiprotonic atomic systems or of (radioactive) nuclei as well as on the time-dependent quantum dynamics of correlated systems. Fundamental tests include, e.g. CPT tests by high-resolution spectroscopy of the 1s-2s transition or of the ground-state hyperfine structure of antihydrogen as well as gravity experiments with antimatter. Structure measurements extend from few-particle QED, correlation, and relativistic effects in antiprotonic atoms to the investigation of the low-energy limit, i.e. of non-perturbative QCD and to nuclear skins exploration.

In addition, low-energy antiprotons are the ideal and perhaps the only tool to study in detail correlated quantum dynamics of few-electron systems in the femto and sub-femtosecond time regime [1,2]. Advanced storage ring and detection technologies in combination will enable, for the first time, access to kinematically complete antiproton-induced rearrangement and fragmentation measurements. These will allow to ultimately benchmark contradicting predictions of existing theories and to explicitly address the few-body Coulomb problem one of the most fundamental, yet unsolved problems in physics.

To enable the efficient investigation of essentially all of these important questions in detail, a novel electrostatic cooler synchrotron and a state-of-the-art in-ring spectrometer are under development in close collaboration between the QUASAR group (http://www.quasar-group.org), the Max-Planck Institute for Nuclear Physics, the GSI Atomic Physics Division, and groups from the University of Heidelberg with the aim of slowing down antiprotons as well as possibly highly charged ions (up to bare uranium) to low energies between 20 and $300 \mathrm{keV} / \mathrm{q}$. This will provide world-wide unique conditions for both in-ring studies with an intensity of up to $10^{12}$ cooled and stored antiprotons or highly charged ions per second, as well as for experiments requiring extracted slow beams and will therefore push the limits in all fields concerned.

For the USR installed in FLAIR, new and challenging techniques need to be developed to ensure multi-user operation and pave the way for a true multi-purpose facility:

- Combination of the electrostatic storage mode with beam deceleration from 300 $\mathrm{keV} / \mathrm{q}$ to $20 \mathrm{keV} / \mathrm{q}$;

- Availability of fast and slow extraction to enable both trap experiments with pulsed beams and nuclear physics type experiments requiring extracted quasiDC beams;

- Electron cooling at all covered energies for a variety of different ions ranging from antiprotons to bare uranium; 
Fig. 1 Schematic layout of the ultra-low energy storage ring. The ring features a four fold symmetry and has a circumference of $42.6 \mathrm{~m}$

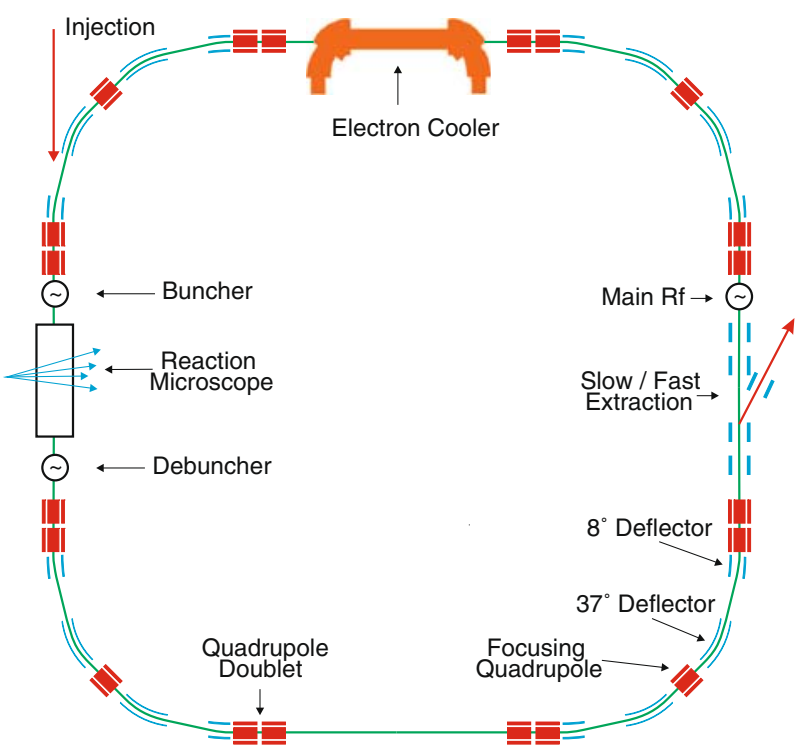

- Particle detection techniques and beam diagnostic elements, operating at cryogenic temperatures and designed for lowest beam energies and currents.

The USR will combine these features for the first time and will thus be one of the essential tools for low-energy physics at FLAIR.

\section{Storage ring layout}

The USR will allow for an efficient deceleration, cooling and storage of antiprotons within FLAIR [3, 4]. In order to be adjustable to the different needs of the experimentalists, the storage ring has to be set to different operation modes. Apart from the basic cooling and deceleration schemes, internal experiments ask for short bunches in the ns-range [5] while external experiments have to be provided with extracted beams of most different pulse duration.

The synchrotron is designed, similar to the initial layout of the USR [6], as a square with four straight sections-each $4 \mathrm{~m}$ in length-which are reserved for extraction, electron cooling and the planned experiments, see Fig. 1. The ring itself has a fourfold symmetry and an overall circumference of $42.6 \mathrm{~m}$. The bending sections of the ring are designed in a novel "split-achromat" geometry. The total deflection of the beam by $90^{\circ}$ in each of the corner sections is realized by a combination of $8^{\circ}-37^{\circ}$ $37^{\circ}-8^{\circ}$ electrostatic cylinder deflectors. For the transverse modulation of the beam quadrupole doublets placed at the entrance and exit of every experimental straight section are used. An additional focusing quadrupole, placed in between the two $37^{\circ}$ deflectors, is included to control the dispersion of the beam. The linear lattice functions for one possible operation mode, providing a round beam suitable for beam cooling and for experiments, are shown in Fig. 2 (left). Due to the special split-achromat lattice, an additional operation mode is possible: In this case, the 
Fig. 2 Overview of the USR lattice in two different modes of operation. Top: Lattice functions with round beam in experiment. Bottom: Lattice function in "zero dispersion" mode
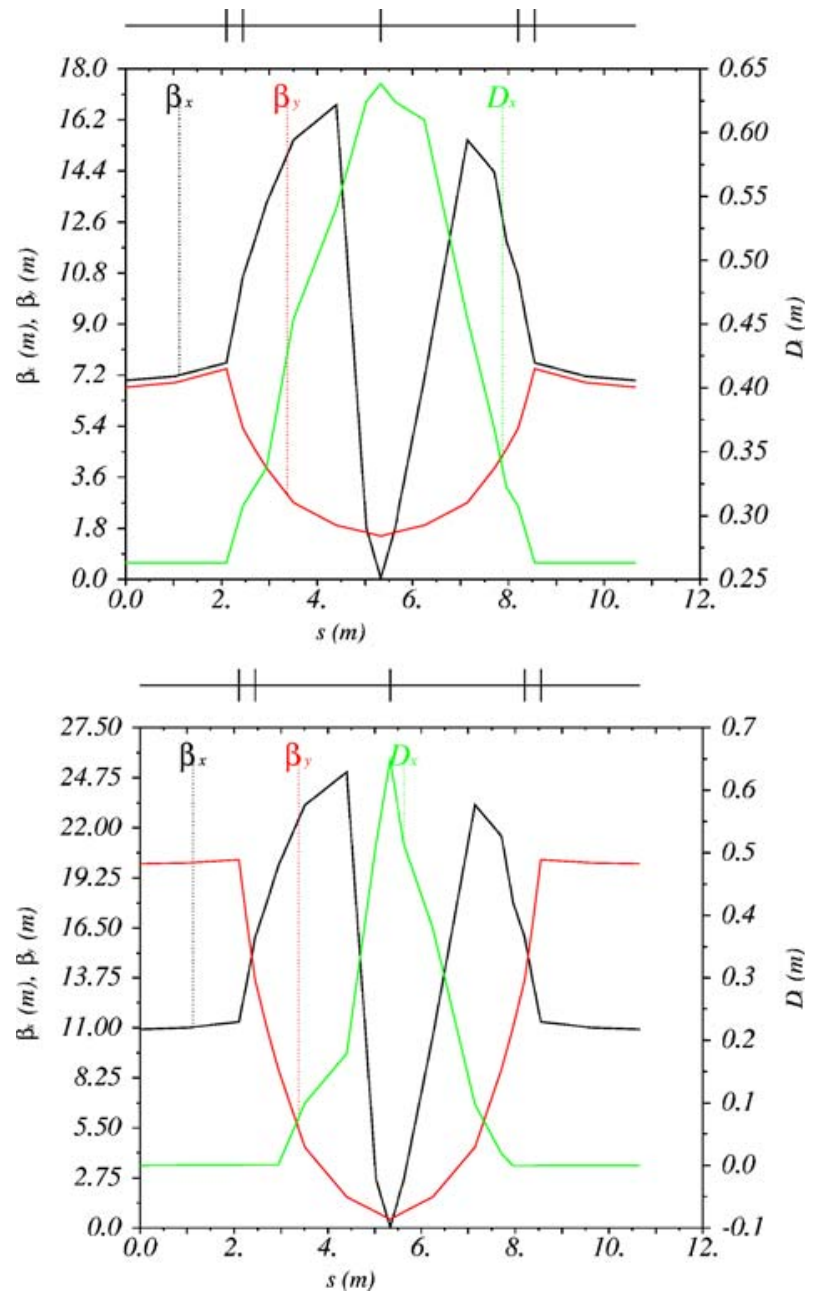

dispersion is reduced to zero in the center of the straight sections, and reaches its maximum value of only $\mathrm{D}_{x}=0.7 \mathrm{~m}$ in the central focusing quadrupole, as shown in Fig. 2 (right). The present machine parameters of the USR are summarized in Table 1.

\subsection{Nanosecond bunches}

In order to reach a high resolution in the envisaged experiments with the novel in-ring reaction microscope, presently being developed in the QUASAR group in collaboration with the Ullrich group at MPIK, ultra-short pulses of $20 \mathrm{keV}$ antiprotons with a time structure of only a few nanoseconds have to be provided. It is clearly impossible to create ultra-short bunches of a few ns duration in one step from a coasting beam: With a revolution period of $20 \mu$ s the required buncher voltage 
Table 1 General parameters of the USR at extraction energy (only antiprotons are considered)

\begin{tabular}{ll}
\hline Energy [keV] & 20 \\
Circumference $[\mathrm{m}]$ & 42.984 \\
Rotation period $[\mu \mathrm{s}]$ & 21.957 \\
Rotation frequency, $\mathrm{F}_{\text {rot }}[\mathrm{kHz}]$ & 45.5436 \\
$\mathrm{RF}$ frequency $\mathrm{F}_{R F}[\mathrm{MHz}]$ & 20.0392 \\
$\mathrm{RF}$ harmonic number $\mathrm{h}_{R F}$ & 440 \\
$\mathrm{RF}$ bucket width [ns] & 49.9 \\
Buncher drift space [cm] & 200 \\
Buncher voltage $[\mathrm{kV}]$ & 0.37 \\
Expected pulse width [ns] & 2 \\
Momentum spread $\left(\right.$ after $\mathrm{e}^{-}$cooling) & $5 * 10^{-4}$ \\
Achromatic mode dispersion $\mathrm{D}_{\max } / \mathrm{D}_{\min }[\mathrm{m}]$ & 0.70 \\
Momentum compaction $\alpha$ & 0.207 \\
Frequency slip factor $\eta$ & -0.8 \\
\hline
\end{tabular}

to provide a $2 \mathrm{~ns}$ time focus would exceed $10 \mathrm{kV}$ and thus the induced energy spread by the phase compression would simply destroy the beam circulating in the ring.

An operation with ultra-short pulses for in-ring experiments might be realized by applying the sequence of the following procedures: Once the beam has been slowed down to $20 \mathrm{keV}$, the coasting beam of antiprotons is cooled down to a momentum spread of $10^{-4}$ [7]. Then the cooled beam is adiabatically captured into $\tau \approx 50 \mathrm{~ns}$ stationary buckets formed by a $20 \mathrm{MHz}$ cavity operating at a high harmonic mode of the ring revolution frequency $\left(\mathrm{h}_{R F}=300-400\right)$.

The desired ultra-short pulses of $\tau=2$ ns duration will then be formed in the symmetry point of the straight section where the reaction microscope is located. The focus will be provided by an additional $3 \beta \lambda / 2$ double drift buncher, placed at the beginning of the straight section. Once the experimental section is crossed, a debuncher will provide phase decompression and limit the growth of the equilibrium momentum spread. Otherwise the increasing energy spread introduced by the phase compressor would cause a beam blow up in the bending sections of the ring. As it was already pointed out, the phase compression will lead to an additional energy spread. This requires that any manipulation of the beam towards short pulses needs to be limited to the straight sections of the ring, where the dispersion function needs to be zero. To allow for this special operation mode, the USR lattice was recently modified substantially [8], as described in the previous section.

\section{Beam diagnostics}

The boundary conditions of the USR project put very high demands on the machines' instrumentation: If one thinks of not only storing antiprotons, but also highly charged ions, ultimate vacuum pressures have to be realized extending to below $10^{-14}$ mbar to ensure reasonable beam life times. Hence, an approach considered in the present study is to cryogenically cool the vacuum chambers of the USR to a temperature of only a few Kelvin. Such a cryogenic electrostatic ion storage ring (Cryogenic Storage Ring CSR) is presently pioneered at the Max Planck Institute for Nuclear Physics [9]; another cryogenic electrostatic storage ring is under construction at the University of 
Stockholm [10]. The extremely low vacuum pressure of the (cooled) USR together with a beam energy of only $20 \mathrm{keV}$ and low currents of singly charged antiprotons between $1 \mathrm{nA}$ and $1 \mu \mathrm{A}$ require the development of new diagnostic methods as most of the standard techniques will no longer work. Different techniques are presently under consideration [11, 12], including ultra-low current pickups, schottky pickups, a cryogenic current comparator, and a novel beam profile monitor based on an extended gas jet curtain $[13,14]$.

\section{Conclusion and outlook}

The ultra-low energy storage ring marks a significant evolution step forward as compared to existing schemes to decelerate antiprotons and possibly highly charged ions to lowest energies. The storage ring layout was adapted to match the requirements of the different users, as well as of specific machine operation conditions, like short bunch mode operation or electron cooling. The chosen split-achromatic geometry promises utmost flexibility while maintaining the compactness and simplicity of the storage ring. In a next step we will analyse the dynamic aperature of the machine in detail, perform real field simulations, and develop field compensation schemes for both the electron cooler as well as the reaction microscope.

Acknowledgements The generous support of the Helmholtz Association of National Research Centers (HGF) under contract number VH-NG-328 and of the Gesellschaft für Schwerionenforschung (GSI) Darmstadt is acknowledged.

Open Access This article is distributed under the terms of the Creative Commons Attribution Noncommercial License which permits any noncommercial use, distribution, and reproduction in any medium, provided the original author(s) and source are credited.

\section{References}

1. Ullrich, J., et al.: Sub-femtosecond correlated dynamics explored with antiprotons. AIP Conf. Proc. 793, 71-88 (2005)

2. Welsch, C.P., et al.: Exploring sub-femtosecond correlated dynamics with an ultra-low energy electrostatic storage ring. AIP Conf. Proc. 796, 266-271 (2005)

3. Widmann, E., et al.: FLAIR - Technical Proposal. http://www.oeaw.ac.at/smi/flair/ (2005). Accessed 24 October 2008

4. Welsch, C.P., Ullrich, J.: FLAIR - a facility for low-energy antiproton and ion research. Hyperfine Interact. 172, 71-80 (2006)

5. Papash, A., Welsch, C.P.: On the possibility of realizing shortest bunches in low-energy storage rings. Particle Physics and Nuclei Letters. doi:10.1134/s1547477109030054

6. Welsch, C.P., et al.: An ultra-low-energy storage ring at FLAIR. Nucl. Instrum. Methods A 546, 405-417 (2005)

7. Welsch, C.P., et al.: Cooling rates of the USR as calculated with BETACOOL. AIP Conf. Proc. 821, 397-401 (2006)

8. Schmid, P., et al.: Preliminary design of a highly-flexible extraction scheme for the USR. In: Proc. Europ. Part. Acc. Conf., Genoa, Italy (2008)

9. Zajfman, D., et al.: Physics with colder molecular ions: the Heidelberg Cryogenic storage ring CSR. J. Phys. Conf. Ser. 4, 296c (2005)

10. Rensfelt, K.-G., et al.: DESIREE - a double electrostatic storage ring. In: Proc. European Part. Acc. Conf., Lucerne, Switzerland (2004)

11. Sieber, T., et al.: A beam diagnostics system for the Heidelberg cryogenic storage ring CSR. In: Proc. European Part. Acc. Conf., Edinburgh, Scotland (2006) 
12. Harasimowicz, J., et al.: Beam instrumentation for the future ultra-low energy electrostatic storage ring at FLAIR. Hyperfine Interact. (2009). doi:10.1007/s10751-009-0047-0

13. Kühnel, K.-U., et al.: A novel beam profile monitor based on a supersonic gas jet. In: Proc. Europ. Part. Acc. Conf., Genoa, Italy (2008)

14. Putignano, M., et al.: Influence of skimmer geometry on a gas-jet curtain for use in a novel beam profile monitor. Hyperfine Interact. (2009, in press) 